\title{
DETERMINATION OF ANTIOXIDANT ADDITIVES IN FOODSTUFFS BY DIRECT MEASUREMENT OF GOLD NANOPARTICLE FORMATION USING RESONANCE LIGHT SCATTERING DETECTION
}

\author{
A. Andreu-Navarro, J.M. Fernández-Romero, A. Gómez-Hens \\ Department of Analytical Chemistry \\ University of Córdoba \\ Campus of Rabanales \\ Annex to Marie Curie building \\ 14071-Córdoba. Spain \\ E-mail: qa1gohea@uco.es \\ Web: http://www.uco.es/FQM-303/
}

\begin{abstract}
The capability of antioxidant compounds to reduce gold(III) to gold nanoparticles (AuNPs) has been kinetically studied in the presence of cetyltrimethylammonium bromide using stopped-flow mixing technique and resonance light scattering as detection system. This study has given rise to a simple and rapid method for the determination of several synthetic and natural antioxidant used as additives in foodstuff samples. The formation of AuNPs was monitored by measuring the initial reaction-rate of the system in about $5 \mathrm{~s}$, using an integration time of $0.1 \mathrm{~s}$. Dynamic ranges of the calibration graphs and detection limits, obtained with standard solutions of the analytes, were $\left(\mu \mathrm{mol} \mathrm{L}{ }^{-1}\right)$ : gallic acid $(0.04-$ $0.59,0.01)$, propyl gallate $(0.04-1.41,0.01)$, octyl gallate $(0.03-0.35,0.08)$, dodecyl gallate $(0.02-$ $0.30,0.007)$, butylated hydroxyanisol $(0.07-0.39,0.009)$, butylated hydroxytoluene $(0.04-0.32,0.01)$, ascorbic acid $(0.11-1.72,0.03)$ and sodium citrate $(0.07-1.29,0.02)$. The regression coefficients were higher than 0.994 in all instances. The precision of the method, expressed as RSD\%, was established at two concentration levels of each analyte, with values ranging between 0.6 and $4.8 \%$. The practical usefulness of the developed method was demonstrated by the determination of several antioxidant additives in foodstuff samples, which were extracted, appropriately diluted and assayed, obtaining recoveries between 95.4 and $99.5 \%$. The results obtained were validated using two reference methods.
\end{abstract}

